\title{
Comparison between PET template-based method and MRI-based method for cortical quantification of florbetapir (AV-45) uptake in vivo
}

\author{
L. Saint-Aubert • F. Nemmi • P. Péran • E. J. Barbeau • \\ P. Payoux • F. Chollet • J. Pariente
}

Received: 28 August 2013 / Accepted: 28 November 2013 / Published online: 19 December 2013

(C) The Author(s) 2013. This article is published with open access at Springerlink.com

\begin{abstract}
Purpose Florbetapir (AV-45) has been shown to be a reliable tool for assessing in vivo amyloid load in patients with Alzheimer's disease from the early stages. However, nonspecific white matter binding has been reported in healthy subjects as well as in patients with Alzheimer's disease. To avoid this issue, cortical quantification might increase the reliability of AV-45 PET analyses. In this study, we compared two quantification methods for AV-45 binding, a classical method relying on PET template registration (route 1), and a MRIbased method (route 2) for cortical quantification.

Methods We recruited 22 patients at the prodromal stage of Alzheimer's disease and 17 matched controls. AV-45 binding was assessed using both methods, and target-to-cerebellum mean global standard uptake values (SUVr) were obtained for each of them, together with SUVr in specific regions of interest. Quantification using the two routes was compared
\end{abstract}

Saint-Aubert L and Nemmi F. contributed equally to this work.

Electronic supplementary material The online version of this article (doi:10.1007/s00259-013-2656-8) contains supplementary material, which is available to authorized users.

L. Saint-Aubert $(\bowtie) \cdot$ F. Nemmi $\cdot$ P. Péran $\cdot$ P. Payoux $\cdot$ F. Chollet $\cdot$ J. Pariente

Inserm, imagerie cérébrale et handicaps neurologiques UMR 825, Centre Hospitalier Universitaire de Toulouse, Pavillon Baudot CHU Purpan lace Dr Baylac, 31059 Toulouse, France

e-mail: laure.saint-aubert@inserm.fr

L. Saint-Aubert $\cdot$ F. Nemmi $\cdot$ P. Péran $\cdot$ P. Payoux $\cdot$ F. Chollet $\cdot$ J. Pariente

Université de Toulouse, UPS, imagerie cérébrale et handicaps neurologiques UMR 825, Centre Hospitalier Universitaire de Toulouse, Toulouse, France between the clinical groups (intragroup comparison), and between groups for each route (intergroup comparison). Discriminant analysis was performed.

Results In the intragroup comparison, differences in uptake values were observed between route 1 and route 2 in both groups. In the intergroup comparison, AV-45 uptake was higher in patients than controls in all regions of interest using both methods, but the effect size of this difference was larger using route 2 . In the discriminant analysis, route 2 showed a higher specificity $(94.1 \%$ versus $70.6 \%$ ), despite a lower sensitivity ( $77.3 \%$ versus $86.4 \%$ ), and D-prime values were higher for route 2 .

Conclusion These findings suggest that, although both quantification methods enabled patients at early stages of Alzheimer's disease to be well discriminated from controls, PET template-based quantification seems adequate for clinical use, while the MRI-based cortical quantification method led to
Université de Toulouse, UPS, centre de recherche cerveau et cognition, France, CNRS, CerCo, Toulouse, France

E. J. Barbeau $\cdot$ F. Chollet $\cdot$ J. Pariente

Service de neurologie, pôle neurosciences, Centre Hospitalier

Universitaire de Toulouse, Toulouse, France

P. Payoux

Service de Médecine Nucléaire, Pôle Imagerie, Centre Hospitalier Universitaire de Toulouse, Toulouse, France 
greater intergroup differences and may be more suitable for use in current clinical research.

Keywords Alzheimer's disease · Florbetapir · Amyloid · PET imaging $\cdot$ Cortex

\section{Introduction}

Alzheimer's disease (AD) is a major current public health issue and has been the target of numerous clinical studies in recent decades to improve its diagnosis from the earliest stages, and to develop new therapies to slow its progression. The diagnosis of probable $A D$ relies on clinical criteria that are constantly being updated $[1,2]$. Early diagnosis of $\mathrm{AD}$, in particular at the prodromal stage - when the first symptoms appear - is now also possible in clinical research thanks to established research criteria based on multimodal assessments. These include amyloid pathology assessment using various methods, such as cerebrospinal fluid (CSF) sampling or amyloid-specific imaging by PET $[2,3]$. Several amyloid ligands have been studied in vivo, two of which are widely used in current clinical research: $\left[{ }^{11} \mathrm{C}-\right] \mathrm{PiB}$ [4] and $\left[{ }^{18} \mathrm{~F}-\right]$ Florbetapir (AV45) [5]. Both have now been proven to be reliable tools for assessing the amyloid burden in the brain of patients with AD compared to healthy controls [6-11]. Our group has recently published a study on AV-45 binding in patients with prodromal $\mathrm{AD}$ [12], and a case report showing amyloid pathology using AV-45 PET imaging during presymptomatic stages of $\mathrm{AD}$ [13].

As the present research criteria include amyloid biomarkers, and now that the Food and Drug Administration [14] and the European Medicines Agency (http:// www.ema.europa.eu/) have approved the use of AV-45 imaging for clinical purposes [15], it is important to seek out the most reliable method for analysing AV-45 imaging. Most research studies have investigated AV-45 uptake using quantification methods based on target-to-cerebellum standard uptake values (SUVr) $[5,8,16]$. Nonspecific AV-45 binding has been reported in white matter [9]. This nonspecific binding may affect AV-45 quantification when using whole-brain analyses. To avoid this issue, a few studies have started to focus on AV-45 binding in grey matter only $[17,18]$. In their study, Rodrigue et al. excluded white matter from their analyses by using AV-45 images from young healthy subjects as masks assuming that no specific binding would be found in a young healthy population [18]. In another recent study, La Joie et al. applied a grey matter mask to AV-45 images that were normalized in MNI space [17].

In the present study, we compared two methods for AV-45 cortical quantification, the first one quantifying regional mean
SUVr measured using an AV-45 template, and the second an optimized method based on cortical signal extraction of SUVr using individually segmented T1 MRI sequences.

\section{Materials and methods}

\section{Participants}

All participants gave their written informed consent. The study was approved by the local ethics committee (Comité de Protection des Personnes Sud-Ouest et Outre-Mer I) and the French Agency for the Safety and Security of Medical Devices (Agence Française de Sécurité Sanitaire des Produits de Santé, reference A90605-58).

For this study, patients over 65 years old and with $\mathrm{AD}$ at the prodromal stage [3] were recruited. They were all recruited from the outpatient memory clinic (Neurology Department, University Hospital, Toulouse, France). Matched cognitively normal $(\mathrm{CN})$ subjects were recruited from among patients' relatives or using recruitment posting in public places. All participants underwent a neuropsychological and a medical assessment, brain MRI, FDG PET and AV-45 PET imaging. CSF sampling was also performed in patients only. To be included in the study, patients had to show preserved autonomy in daily life (Clinical Dementia Rating $\leq 0.5$ ) but a memory complaint of more than 6 months duration attested to by isolated memory impairment on neuropsychological assessment [19] and one or more of the following features:

- Medial temporal lobe atrophy assessed on MRI scan (sequences detailed below)

- Temporoparietal hypometabolism pattern on cerebral FDG PET scan (classical clinical acquisitions)

- Abnormal CSF biomarkers according to published criteria $[20,21]$

Patients were not included if they had a concomitant neurological or psychiatric disease, or if they were affected by any clinically significant pathology that could explain the memory complaint. Significant white matter hyperintensities found on T2-weighted MRI images were a reason for exclusion.

$\mathrm{CN}$ subjects were included if they had no memory complaint, no neurological or psychiatric disease history, and if they had no first-degree relatives with AD. They were excluded if they showed significant white matter hyperintensities on T2-weighted MR images or any cognitive impairment on neuropsychological assessment.

More details on this population recruitment have been published elsewhere [12]. 
MRI and AV-45 acquisitions

\section{Brain MRI scans}

MRI scans were performed in all participants using a 3-T imager (Intera Achieva; Philips, Best, The Netherlands). High-resolution anatomical images using a 3-D T1-weighted sequence (in-plane resolution $1 \times 1 \mathrm{~mm}$, slice thickness $1 \mathrm{~mm}$, repetition time/echo time/inversion time $8,189 / 3.75$ / $1,012.2 \mathrm{~ms}$, flip angle $8^{\circ}$, field of view $240 \times 256$, and 160 contiguous slices) and a T2-weighted sequence (reconstructed resolution $0.45 \times 0.45 \times 3 \mathrm{~mm}$, repetition time/echo time $4,132 / 80 \mathrm{~ms}$, flip angle $90^{\circ}$, field of view $240 \times 184$, and 43 slices) were obtained. $\mathrm{CN}$ subjects underwent the same MRI acquisitions as patients.

\section{AV-45 PET scans}

All participants underwent a PET scan using AV-45 amyloid marker. All scans were performed on the same hybrid PET/CT scanner (Biograph 6 TruePoint Hirez; Siemens Medical Solutions) using $3-\mathrm{D}$ detection mode producing images with voxels of $1 \times 1 \times 1.5 \mathrm{~mm}$ and a spatial resolution of approximately $5 \mathrm{~mm}$ full-width at half-maximum at the centre of the field of view). Both CT and PET scans were acquired. Cerebral emission scans were started $50 \mathrm{~min}$ after injection of 3.7 MBq/kg weight of AV-45 and lasted for $20 \mathrm{~min}$. PET data were corrected for partial volume effects using the point spread function (PSF) modelling implemented by Siemens (HD-PETC). Briefly the manufacturer previously measured the response of a point source from many points in its FOV and incorporated measured PSFs in the reconstruction algorithms. Using measured PSFs, HD-PET effectively positions the lines of response in their actual geometric location, which reduces blurring and distortion in the final images.

Three independent raters (two nuclear medicine physicians and one radiologist physicist) with extensive experience reading AV-45 PET scans, blind to clinical information, examined all PET scans. A two-point rating scale (0 normal, 1 amyloidpositive) was used. AV-45 PET scans were considered amyloid-positive when the rating score was 1 for at least two raters. The maximum delay between the two imaging examinations was 3 months.

Image processing

\section{Route 1: Template-based AV-45 quantification}

AV-45-PET scans were linearly registered with 12 degrees of freedom (DOF) and trilinear interpolation onto a template from Avid (http://www.avidrp.com/), which is in the Montreal Neurological Institute (MNI) space, using FLIRT (FSL library tools) [22].
Regional cortical AV-45 mean SUV was measured in each subject using a Matlab (The MathWorks ${ }^{\circledR}$ ) script developed in-house (Fig. 1a). Mean global SUVs, SUVs in the five lobes, and SUVs in specific regions of interest (ROIs: orbitofrontal, anterior cingulate, posterior cingulate and precuneus) were calculated. These regions were selected as they have shown different $\mathrm{AV}-45$ binding in studies comparing $\mathrm{AD}$ patients to $\mathrm{CN}$ subjects $[8,9,12,16,23]$. These regions were defined using the anatomical automatic labelling (AAL) atlas [24] provided by MRIcron software, that was linearly registered (12 DOF, trilinear interpolation) onto the AV-45 template and binarized. To minimize the bias due to nonspecific white matter binding, the AAL atlas was masked for grey matter. To do so, we used the grey matter probability map from SPM8 (Wellcome Trust Centre for Neuroimaging, London, UK), that was binarized using a threshold of 0.3 - meaning that only voxels with a probability greater than $30 \%$ of being grey matter were selected.

SUVs were then normalized (SUVr) to the whole cerebellar mean uptake (vermis excluded) using Matlab and pooled by group for statistical comparison (Fig. 1a).

\section{Route 2: Cortical MRI-based AV-45 quantification}

Grey matter was segmented from the 3-D T1 anatomical image of each participant using FAST and FIRST (FSL library tools), and the resulting grey matter mask was binarized using a threshold of 0.3 - as used for route 1 [25]. Then, for each subject, the CT scan obtained during PET data acquisition was linearly registered (12 DOF) onto the relative T1 anatomical image. The transformation matrix obtained was applied to the AV-45 image of the subject so that the AV-45 image was in the T1 space. The grey matter mask from T1 segmentation (see above) was applied to the transformed AV-45 image. The AAL atlas [24] was also registered from MNI space to each individual T1 space. To do this, the linear transformation matrix from the registration of each single subject's T1 onto MNI space was first calculated. The inverse of this matrix was then applied for each subject to the AAL atlas to obtain ROIs in the subject's space. Finally, the registered atlases were binarized.

The cerebral regions studied were the same as those studied in route 1. Values of regional cortical AV-45 mean SUV were obtained for each subject using the same Matlab script as in route 1 (Fig. 1b). SUVs were normalized (SUVr) to the whole cerebellar mean uptake (vermis excluded, identical definition to that used in route 1) and pooled by group for statistical comparison.

\section{Statistical analyses}

The threshold for significance was set at $p<.05$. For the neuropsychological assessments, groups were compared using the Mann-Whitney or chi-squared statistical tests. A 


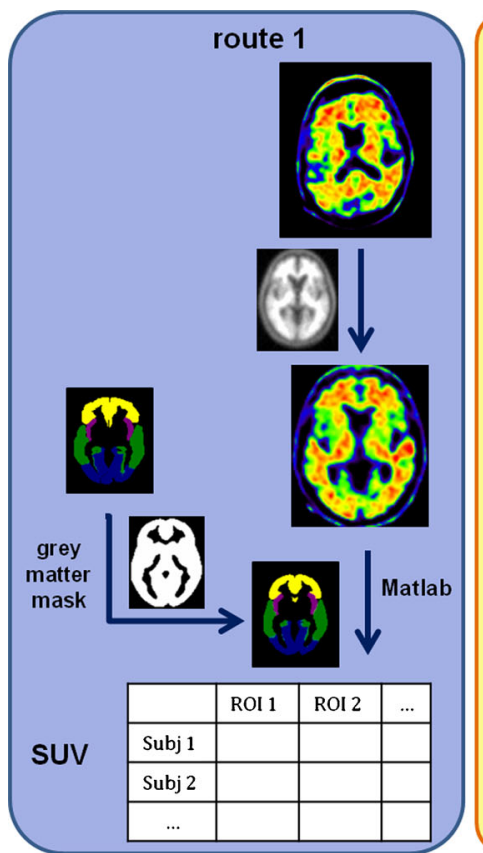

Fig. 1 AV-45 quantification procedures: a cortical PET template-based (route 1) and b cortical MRI-based (route 2). a In route 1, AV-45 acquisitions were linearly registered onto a template from Avid (http:// www.avidrp.com/), and regional uptake was quantified for each subject in the MNI space using the AAL atlas [24] that was masked with a grey matter probability map. Uptake values were collected using Matlab. b In route 2 , for each subject, the CT scan acquired together with the AV-45 image was registered onto the MRI anatomical space defined by the T1

parametric paired $t$ test with Bonferroni-Holmes correction for multiple comparisons was used to compare SUVr values between the two routes for both groups and to compare regional uptake differences between groups for both quantification methods. The effect sizes were assessed using Cohen's $d$ [26]. Standard deviations were weighted by sample size as the number of participants was not equal between groups [27].

For discriminant analysis of the imaging data from both methods, receiver operating characteristics analyses were performed on the mean global SUVr values to study the diagnostic power. The areas under the curve (AUC) were calculated and sensitivity and specificity were computed at the optimal cut-off points. D-prime values as well as their associated bias measures $\mathrm{C}$ were also computed for several cut-off values: the value leading to the same sensitivity and specificity for both routes, the value leading to the best sensitivity and specificity trade-off for route 1 , and the value leading to the best sensitivity and specificity trade-off for route 2 .

\section{Results}

Twenty-two patients with prodromal $\mathrm{AD}$ and $17 \mathrm{CN}$ subjects were included in the study (see supplementary data 1 for individual profiles on research criteria). The two groups were

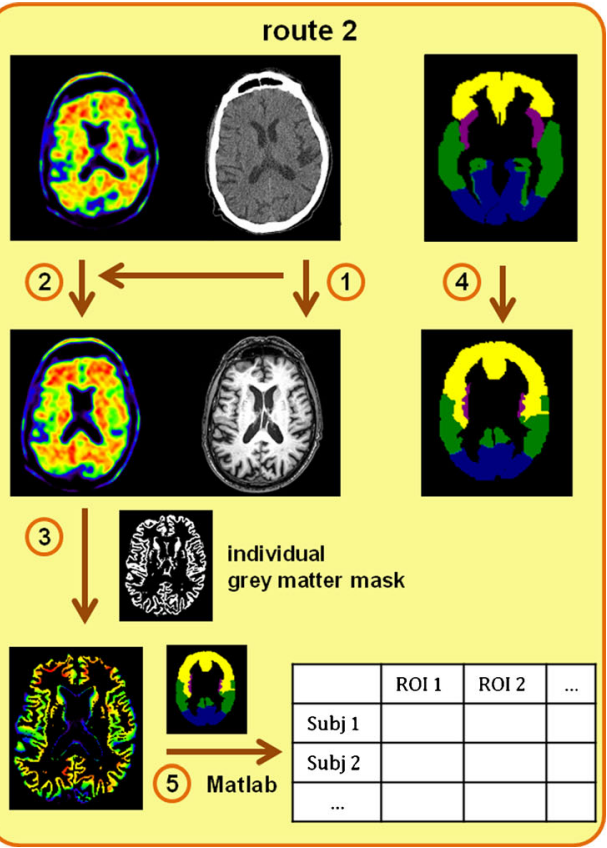

image of the subject concerned (1). The transformation matrix obtained was then applied to the AV-45 image of the subject so that the AV-45 image was in the T1 space (2). A binarized grey matter mask obtained from MRI segmentation was applied to the transformed AV-45 image (3). The AAL atlas was also registered onto each individual T1 space using the inverse of the transformation matrix from the $\mathrm{T} 1$ registration onto MNI space (4). Regional cortical uptake was collected for each subject using Matlab (5). Arrows represent transformation (Subj subject)

matched for age and level of education (see Table 1 for clinical and neuropsychological data).

Visual assessment of AV-45 PET scans revealed amyloidpositive profiles in 18 patients out of 22 , and in $2 \mathrm{CN}$ subjects out of 17 .

Table 1 Clinical and neuropsychological assessment

\begin{tabular}{|c|c|c|c|}
\hline & $\begin{array}{l}\text { Prodromal } \\
\text { patients } \\
(n=22)\end{array}$ & $\begin{array}{l}\text { CN subjects } \\
(n=17)\end{array}$ & $p$ value \\
\hline Age (years) & $72.4 \pm 5.0$ & $69.9 \pm 4.8$ & .110 \\
\hline Gender (M/F) & $12 / 10$ & $7 / 10$ & .408 \\
\hline Level of education (years) & $11.3 \pm 2.7$ & $12.8 \pm 3.3$ & .163 \\
\hline Disease duration (years) & $3.8 \pm 3.6$ & - & - \\
\hline $\begin{array}{l}\text { Daily-life autonomy } \\
\text { (CDR scale) }\end{array}$ & $0.5 \pm .0$ & $0.0 \pm .0$ & $.001^{*}$ \\
\hline Global cognitive state (MMSE) & $25.7 \pm 1.4$ & $28.4 \pm .7$ & $<.001^{*}$ \\
\hline \multicolumn{4}{|c|}{ Anterograde verbal memory (FCSRT test) } \\
\hline Sum of free recalls $(/ 48)$ & $11.6 \pm 5.9$ & $32.2 \pm 4.6$ & $.001^{*}$ \\
\hline $\begin{array}{l}\text { Sum of free and cued } \\
\text { recalls }(/ 48)\end{array}$ & $28.7 \pm 11.9$ & $46.6 \pm 1.9$ & $.001 *$ \\
\hline
\end{tabular}

CDR Clinical Dementia Rating, FCSRT Free and Cued Selective Recall Reminding test

$* p<.05$ 
Table 2 Comparison between the two quantification methods for both groups. AV-45 mean global and target-to-cerebellum SUVr values and associated standard deviations are shown for the two groups using PET templatebased quantification (route 1 ) and MRI-based quantification (route $2)$. Significant $p$ values $(<.05)$ are shown

\begin{tabular}{|c|c|c|c|c|c|c|}
\hline \multirow[t]{2}{*}{ Region } & \multicolumn{3}{|l|}{ Patients } & \multicolumn{3}{|c|}{$\mathrm{CN}$ subjects } \\
\hline & Route 1 & Route 2 & $p$ value & Route 1 & Route 2 & $p$ value \\
\hline Global & $1.47 \pm .24$ & $1.50 \pm .32$ & - & $1.25 \pm .13$ & $1.18 \pm .09$ & .010 \\
\hline Frontal lobes & $1.49 \pm .28$ & $1.54 \pm .38$ & - & $1.29 \pm .17$ & $1.19 \pm .13$ & .010 \\
\hline Temporal lobes & $1.42 \pm .22$ & $1.45 \pm .30$ & - & $1.19 \pm .13$ & $1.16 \pm .07$ & - \\
\hline Insular lobes & $1.37 \pm .27$ & $1.46 \pm .32$ & .045 & $1.14 \pm .13$ & $1.19 \pm .11$ & - \\
\hline Parietal lobes & $1.54 \pm .25$ & $1.51 \pm .35$ & - & $1.26 \pm .14$ & $1.15 \pm .09$ & .002 \\
\hline Occipital lobes & $1.43 \pm .22$ & $1.49 \pm .31$ & - & $1.24 \pm .13$ & $1.22 \pm .08$ & - \\
\hline Orbitofrontal & $1.47 \pm .31$ & $1.60 \pm .41$ & .011 & $1.22 \pm .17$ & $1.23 \pm .16$ & - \\
\hline Anterior cingulate & $1.59 \pm .31$ & $1.67 \pm .41$ & - & $1.36 \pm .20$ & $1.30 \pm .16$ & - \\
\hline Posterior cingulate & $1.82 \pm .31$ & $1.74 \pm .36$ & .021 & $1.55 \pm .27$ & $1.36 \pm .14$ & .005 \\
\hline Precuneus & $1.56 \pm .30$ & $1.56 \pm .36$ & - & $1.20 \pm .14$ & $1.14 \pm .09$ & - \\
\hline
\end{tabular}

Comparison of SUVr values between the two routes for both groups

In the patient group, regional SUVr values in the insula and the orbitofrontal region were significantly lower using route 1 than route 2, while SUVr values in the posterior cingulate were significantly higher using route 1 . In the $\mathrm{CN}$ group, mean global as well as frontal, parietal and posterior cingulate $\mathrm{SUVr}$ values were significantly higher using route 1 than route 2 (Table 2).

Imaging comparison between groups

Both methods showed significantly higher AV-45 uptake in the patient group than in the $\mathrm{CN}$ subjects in all ROIs.

\section{Route 1 (AV-45 template-based quantification)}

Compared to the $\mathrm{CN}$ subjects, the patients showed a significantly higher uptake of AV-45 in the whole brain $(p=.002)$, in every cerebral lobe (frontal $p=.013$, parietal $p=.001$, temporal $p=.003$, insula $p=.007$, occipital $p=.008$ ) and each ROI (orbitofrontal $p=.018$, anterior cingulate $p=.010$, posterior cingulate $p=.014$, precuneus $p<.001$; Fig. 2a).

\section{Route 2 (MRI-based AV-45 quantification)}

Compared to the $\mathrm{CN}$ group, the patients group showed a significantly higher uptake of AV-45 in the whole cortex $(p<.001)$, in every cerebral lobe (frontal $p=.002$, parietal $p=.001$, temporal $p=.001$, insula $p=.002$, occipital $p=.003$ ), and each ROI (orbitofrontal $p=.002$, anterior cingulate $p=.001$, posterior cingulate $p=.001$, precuneus $p<.001$; Fig. 2b).

\section{Effect size}

AV-45 uptake was higher in patients than in $\mathrm{CN}$ subjects with both methods. Cohen's $d$ values were calculated for all regions. They were always higher with route 2 than with route 1 (Fig. 2). route 1
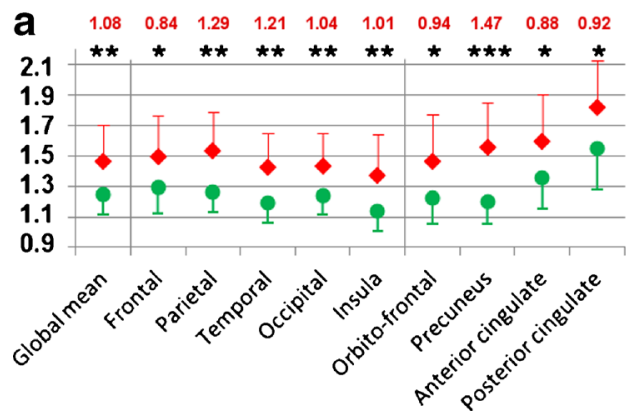

Fig. 2 Target-to-cerebellum cortical AV-45 uptake ratios using a route 1 (the PET template-based method) and $\mathbf{b}$ route 2 (the MRI-based method). Mean values in all ROIs are shown with associated standard deviations route 2

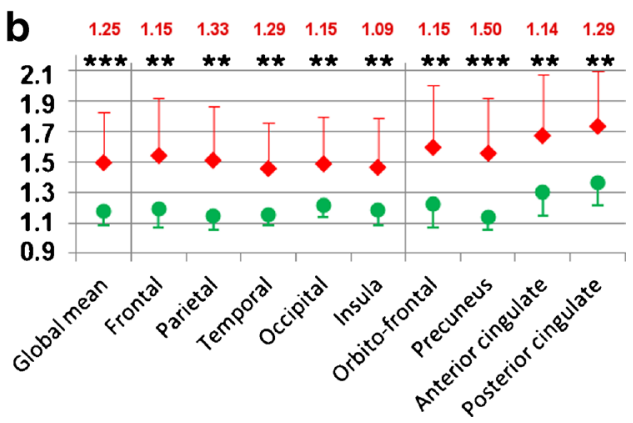

(red diamonds patients, green circles control subjects) ${ }^{*} p<.05,{ }^{* *} p<.01$, $* * * p<.001$. Cohen's $d$ values for each region are given in red characters 
Discriminant analysis

Discriminant analysis of the mean global AV-45 SUVr values from route 1 showed the highest sensitivity $(86.4 \%)$ and specificity $(70.6 \%)$ with a cut-off value of 1.67 (AUC 0.81). For this cut-off value, D-prime was 1.64 for route 1 and 2.31 for route 2 (Fig. 3).

In contrast, discriminant analysis of the mean global AV-45 SUVr values from route 2 showed the highest sensitivity $(77.3 \%)$ and specificity (94.1\%) with a cut-off value of 1.28 (AUC 0.81). For this cut-off value, D-prime was 1.32 for route 1 and 2.31 for route 2 (Fig. 3).

\section{Discussion}

In this study, we compared two quantitative methods for assessing cortical AV-45 target-to-cerebellum uptake in a population of patients with prodromal AD matched to $\mathrm{CN}$ subjects. Route 1 relied on cortical quantification using a standardized PET template. Route 2 relied on cortical uptake using cortical signal extraction from individual MRI sequences. We showed that (1) there were significant differences between the SUVr values from route 1 and route 2 in the patients and $\mathrm{CN}$ groups; (2) global quantification relying on the cortex only (route 2) showed a greater difference between SUVr of prodromal patients and SUVr of CN subjects than the template-based registration (route 1). To our knowledge, this is the first study showing significant differences between PET template-based and MRI-based cortical AV-45 quantification methods.
Patients were included if they were diagnosed with prodromal AD according to research criteria [3]. Amyloid burden is thought to appear early, before the first clinical symptoms, and to have reached a high level at the prodromal stage. In this study, both methods showed significantly higher AV-45 uptake in prodromal patients than in matched $\mathrm{CN}$ subjects, both global uptake and uptake in all neocortical regions.

To avoid interference from white matter uptake in our quantification methods, we used grey matter masks. However, the same mask was used for all PET images in route 1, all being in MNI space, while a specific grey matter mask was used for each participant derived from segmentation of the subject's own MRI scan in route 2, potentially leading to more precision in cortical quantification. This may be the reason for significant differences observed between the two routes. In the $\mathrm{CN}$ group there were significant decreases in global uptake and in frontal, parietal and posterior cingulate uptakes using route 2 compared to route 1 . It is known that $\mathrm{CN}$ subjects show important nonspecific AV-45 binding in white matter, which may affect whole-brain quantification. Our PET template-based quantification method (route 1) may have only partially suppressed the effect of white matter uptake, while the individual MRI-based method (route 2) produced lower cortical SUVr values in CN subjects. Similar results were found for the posterior cingulate region in the patient group, with significantly lower uptake found using route 2 . One hypothesis that may be proposed is that the white matter PET signal could easily spill over onto the posterior cingulate because of the latter's proximity to dense white matter fibres. As in the CN subjects, the MRI-based method would minimize the effect of white matter uptake in this region in patients. We also found significant increases in the uptake values
Fig. 3 Receiver operating characteristics curves for route 1 and route 2 (a orange curve route 1 , green curve route 2 ). b-d Dprime values and the corresponding values of the bias measure $\mathrm{C}$ for three different SUVr values: $b$ cut-off value for same sensitivity/specificity for route 1 and route 2 , c cut-off value for best sensitivity/ specificity for route $1, \mathbf{d}$ cut-off value for best sensitivity/ specificity for route 2

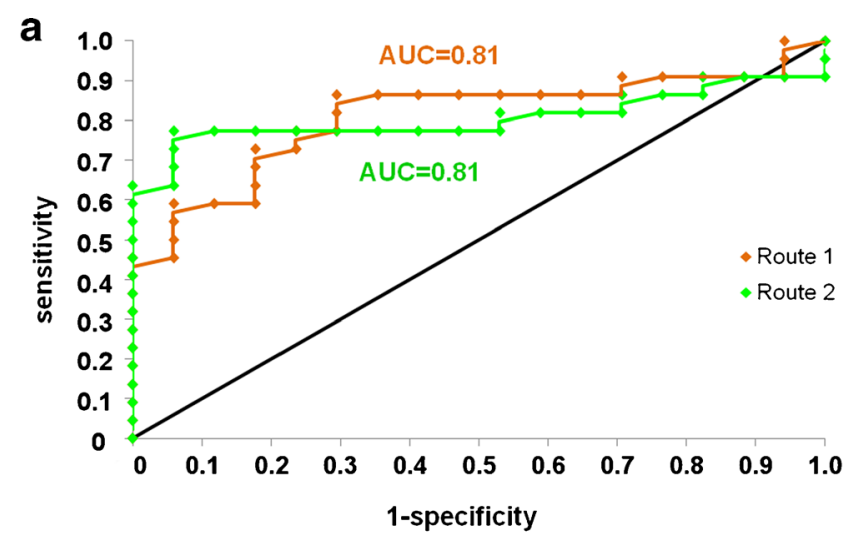

\begin{tabular}{l|c|c|}
\multicolumn{2}{|c}{$b$} \\
\cline { 2 - 3 } \multicolumn{1}{l|}{} & route 1 & route 2 \\
\hline cut-off SUVr & 1.38 & 1.25 \\
\hline specificity & $77 \%$ & $77 \%$ \\
\hline sensitivity & $76 \%$ & $76 \%$ \\
\hline D-prime & 1.47 & 1.47 \\
\hline C & 0.01 & 0.01 \\
\hline
\end{tabular}

\begin{tabular}{|c|c|c|}
\hline & route 1 & route 2 \\
\hline cut-off SUVr & 1.28 & 1.28 \\
\hline specificity & $86 \%$ & $77 \%$ \\
\hline sensitivity & $59 \%$ & $94 \%$ \\
\hline D-prime & 1.32 & 2.31 \\
\hline C & 0.44 & -0.41 \\
\hline
\end{tabular}


in the insula and the orbitofrontal regions of patients when using route 2 . This may be due to high specific amyloid binding that would be reduced by some white matter signal "surviving" in route 1 . Further studies testing such hypotheses are necessary.

We used MRI for individual grey matter segmentation since classical T1-weighted MRI sequences have higher resolution than PET images and since most patients nowadays undergo clinical MRI scans for diagnostic purposes. Many studies on amyloid imaging have used visual assessment to determine the uptake profiles of amyloid markers and to classify subjects as "positive" or "negative". Although it may be the fastest way so far found to assess AV-45 uptake profiles in clinical routine, visual assessment has shown some limitations [28] and should be considered with caution. The use of SUV quantification, and in particular cortical quantification, should provide more precise results. It is thus crucial to develop fast, reproducible techniques.

A recent study investigated similar questions regarding analyses of amyloid PET imaging using PiB in AD patients and $\mathrm{CN}$ subjects [25]. The authors compared a method using a $\mathrm{PiB}$ template for normalization, and one method using MRI for grey matter segmentation and normalization. Both methods included transformation into MNI space - which was not the case in our study. The authors showed that the PET template-based and MRI-based methods were slightly different, with systematically higher, but comparable, values for the PET template-based method in every group. In our study, the results of our two methods were not comparable. Route 2 (MRI-based method) showed greater differences and a larger effect sizes of the cortical SUVr values of prodromal $\mathrm{AD}$ patients and $\mathrm{CN}$ subjects than route 1 . However, we used a different approach to that of Edison et al. regarding the MRIbased method, as we performed our analyses in the individual space in all subjects to stay closest to the individual grey matter anatomy. Such MRI-based cortical quantification would thus lead to greater accuracy in individual regional quantification. Besides, our study used the amyloid biomarker AV-45, which has shown higher nonspecific binding than PiB [16]. This could also partly explain the significant differences we found between route 1 and route 2 that were not found by Edison et al. [25]. In another recent study, Landau et al. investigated amyloid ligand uptake using PiB and AV-45 in a patients with mild cognitive impairment compared to healthy subjects [29]. They used similar methods to our routes 1 and 2 for cortical quantification, and found similar results between the two routes. These results, at odds with ours, might be accounted for by differences in the size of the selected ROIs in the two studies.

On discriminant analysis, the two routes showed different patterns. Route 1 showed a higher sensitivity than route 2 ( $86.4 \%$ versus $77.3 \%$, respectively), but a lower specificity ( $70.6 \%$ versus $94.1 \%$, respectively). The high specificity of route 2 corresponds to only one false-positive result, which is in accordance with results of other studies [10, 30, 31]. This means that a cortical MRI-based quantification gives fewer false-positive results among $\mathrm{CN}$ subjects, and may be more suitable for use in clinical research. On the other hand, the conventional AV-45 template-based method gave fewer falsenegative results among patients. As a consequence, such a method appears adequate for clinical use if the aim is to confirm the diagnosis in patients presenting with other markers suggestive of AD. The D-prime calculation showed higher values for route 2 , suggesting an overall better quality of prediction using MRI-based quantification. Bias measure $\mathrm{C}$ confirmed the tendency of route 1 to be sensitive, as opposed to the tendency of route 2 to be specific. Of note, visual assessment showed discrimination results in between, with a sensitivity of $81.8 \%$ and a specificity of $88.2 \%$.

\section{Conclusion}

This study demonstrated that both cortical MRI-based and PET template-based AV-45 quantifications efficiently discriminate between prodromal patients and healthy subjects. Clinical diagnosis may rely on PET template-based quantification as it discriminates better between patients and healthy subjects and it is faster and cheaper to perform than MRIbased quantification. On the other hand the latter would be more suitable for research purposes, as only 1 out of $17 \mathrm{CN}$ subjects was misclassified. A further AV-45 quantification study including a larger sample should be performed.

Acknowledgments This study was supported by a grant from the University Hospital of Toulouse, local grant 2007, and a grant from the Agence Nationale de la Recherche. The authors thank the promoter of this study, CHU Toulouse, and the clinical manager Delphine Vernet.

Conflicts of interest Prof. P. Payoux received honoraria from serving on the scientific advisory board of Lilly.

Prof. F. Chollet is currently consultant for the "Institut de recherche Pierre Fabre, France"

Prof. J. Pariente serves on the editorial board of the Journal of Alzheimer's Disease, and received grants from the Agence Nationale de la Recherche (French National Research Agency) and Toulouse teaching hospital for this study.

Open Access This article is distributed under the terms of the Creative Commons Attribution License which permits any use, distribution, and reproduction in any medium, provided the original author(s) and the source are credited.

\section{References}

1. McKhann G, Drachman D, Folstein M, Katzman R, Price D, Stadlan EM. Clinical diagnosis of Alzheimer's disease: report of the NINCDS-ADRDA Work Group under the auspices of Department of Health and Human Services Task Force on Alzheimer's Disease. Neurology. 1984;34:939-44. 
2. McKhann GM, Knopman DS, Chertkow H, Hyman BT, Jack Jr CR, Kawas $\mathrm{CH}$, et al. The diagnosis of dementia due to Alzheimer's disease: recommendations from the National Institute on AgingAlzheimer's Association workgroups on diagnostic guidelines for Alzheimer's disease. Alzheimers Dement. 2011;7:263-9. doi:10. 1016/j.jalz.2011.03.005.

3. Dubois B, Feldman HH, Jacova C, Dekosky ST, Barberger-Gateau P, Cummings J, et al. Research criteria for the diagnosis of Alzheimer's disease: revising the NINCDS-ADRDA criteria. Lancet Neurol. 2007;6:734-46. doi:10.1016/S1474-4422(07)70178-3.

4. Klunk WE, Engler H, Nordberg A, Wang Y, Blomqvist G, Holt DP, et al. Imaging brain amyloid in Alzheimer's disease with Pittsburgh Compound-B. Ann Neurol. 2004;55:306-19. doi:10.1002/ana. 20009.

5. Clark CM, Schneider JA, Bedell BJ, Beach TG, Bilker WB, Mintun $\mathrm{MA}$, et al. Use of florbetapir-PET for imaging beta-amyloid pathology. JAMA. 2011;305:275-83. doi:10.1001/jama.2010.2008.

6. Jack Jr CR, Lowe VJ, Senjem ML, Weigand SD, Kemp BJ, Shiung $\mathrm{MM}$, et al. $11 \mathrm{C} \mathrm{PiB}$ and structural MRI provide complementary information in imaging of Alzheimer's disease and amnestic mild cognitive impairment. Brain. 2008;131:665-80. doi:10.1093/brain/ awm336.

7. Tosun D, Schuff N, Mathis CA, Jagust W, Weiner MW. Spatial patterns of brain amyloid-beta burden and atrophy rate associations in mild cognitive impairment. Brain. 2011;134:1077-88. doi:10. 1093/brain/awr044.

8. Fleisher AS, Chen K, Liu X, Roontiva A, Thiyyagura P, Ayutyanont $\mathrm{N}$, et al. Using positron emission tomography and florbetapir F18 to image cortical amyloid in patients with mild cognitive impairment or dementia due to Alzheimer disease. Arch Neurol. 2011;68:1404-11. doi:10.1001/archneurol.2011.150.

9. Wong DF, Rosenberg PB, Zhou Y, Kumar A, Raymont V, Ravert HT, et al. In vivo imaging of amyloid deposition in Alzheimer disease using the radioligand 18F-AV-45 (florbetapir [corrected] F 18). J Nucl Med. 2010;51:913-20. doi:10.2967/jnumed.109.069088.

10. Rowe CC, Ellis KA, Rimajova M, Bourgeat P, Pike KE, Jones G, et al. Amyloid imaging results from the Australian Imaging, Biomarkers and Lifestyle (AIBL) study of aging. Neurobiol Aging. 2010;31:1275-83. doi:10.1016/j.neurobiolaging.2010.04.007.

11. Johnson KA, Sperling RA, Gidicsin CM, Carmasin JS, Maye JE, Coleman RE, et al. Florbetapir (F18-AV-45) PET to assess amyloid burden in Alzheimer's disease dementia, mild cognitive impairment, and normal aging. Alzheimers Dement. 2013;9(5 Suppl):S72-83. doi:10.1016/j.jalz.2012.10.007.

12. Saint-Aubert L, Barbeau EJ, Peran P, Nemmi F, Vervueren C, Mirabel H, et al. Cortical florbetapir-PET amyloid load in prodromal Alzheimer's disease patients. EJNMMI Res. 2013;3:43. doi:10.1186/ 2191-219X-3-43.

13. Saint-Aubert L, Planton M, Hannequin D, Albucher JF, Delisle MB, Payoux P, et al. Amyloid imaging with AV45 ((18)F-florbetapir) in a cognitively normal AbetaPP duplication carrier. J Alzheimers Dis. 2012;28:877-83. doi:10.3233/JAD-2011-111598.

14. FDA approves 18F-florbetapir PET agent. J Nucl Med. 2012;53:15N. doi:53/6/15N [pii]

15. Booij J, Arbizu J, Darcourt J, Hesse S, Nobili F, Payoux P, et al. Appropriate use criteria for amyloid PET imaging cannot replace guidelines: on behalf of the European Association of Nuclear Medicine. Eur J Nucl Med Mol Imaging. 2013;40:1122-5. doi:10. 1007/s00259-013-2415-x.

16. Wolk DA, Zhang Z, Boudhar S, Clark CM, Pontecorvo MJ, Arnold SE. Amyloid imaging in Alzheimer's disease: comparison of florbetapir and Pittsburgh compound-B positron emission tomography. J Neurol Neurosurg Psychiatry. 2012;83:923-6. doi:10.1136/ jnnp-2012-302548.
17. La Joie R, Perrotin A, Barre L, Hommet C, Mezenge F, Ibazizene M, et al. Region-specific hierarchy between atrophy, hypometabolism, and beta-amyloid (Abeta) load in Alzheimer's disease dementia. J Neurosci. 2012;32:16265-73. doi:10.1523/JNEUROSCI.2170-12. 2012.

18. Rodrigue KM, Kennedy KM, Devous Sr MD, Rieck JR, Hebrank AC, Diaz-Arrastia R, et al. beta-Amyloid burden in healthy aging: regional distribution and cognitive consequences. Neurology. 2012;78:387-95. doi:10.1212/WNL. 0b013e318245d295.

19. Sarazin M, Berr C, De Rotrou J, Fabrigoule C, Pasquier F, Legrain S, et al. Amnestic syndrome of the medial temporal type identifies prodromal AD: a longitudinal study. Neurology. 2007;69:1859-67. doi:10.1212/01.wnl.0000279336.36610.f7.

20. Wiltfang J, Esselmann H, Bibl M, Hull M, Hampel H, Kessler H, et al. Amyloid beta peptide ratio $42 / 40$ but not A beta 42 correlates with phospho-Tau in patients with low- and high-CSF A beta 40 load. J Neurochem. 2007;101:1053-9. doi:10.1111/j.1471-4159.2006. 04404.x.

21. Wallon D, Rousseau S, Rovelet-Lecrux A, Quillard-Muraine M, Guyant-Marechal L, Martinaud O, et al. The French series of autosomal dominant early onset Alzheimer's disease cases: mutation spectrum and cerebrospinal fluid biomarkers. J Alzheimers Dis. 2012;30:847-56. doi:10.3233/JAD-2012-120172.

22. Jenkinson M, Bannister P, Brady M, Smith S. Improved optimization for the robust and accurate linear registration and motion correction of brain images. Neuroimage. 2002;17:825-41.

23. Lin KJ, Hsu WC, Hsiao IT, Wey SP, Jin LW, Skovronsky D, et al. Whole-body biodistribution and brain PET imaging with [18F]AV45, a novel amyloid imaging agent - a pilot study. Nucl Med Biol. 2010;37:497-508. doi:10.1016/j.nucmedbio.2010.02.003.

24. Tzourio-Mazoyer N, Landeau B, Papathanassiou D, Crivello F, Etard $\mathrm{O}$, Delcroix N, et al. Automated anatomical labeling of activations in SPM using a macroscopic anatomical parcellation of the MNI MRI single-subject brain. Neuroimage. 2002;15:273-89. doi:10.1006/ nimg.2001.0978.

25. Edison P, Carter SF, Rinne JO, Gelosa G, Herholz K, Nordberg A, et al. Comparison of MRI based and PET template based approaches in the quantitative analysis of amyloid imaging with PIB-PET. Neuroimage. 2013;70:423-33. doi:10.1016/j.neuroimage.2012.12. 014.

26. Cohen J. Statistical power analysis for the behavioral sciences. 2nd ed. Hillsdale, NJ: Lawrence Erlbaum Associates; 1988

27. Zakzanis KK. Statistics to tell the truth, the whole truth, and nothing but the truth: formulae, illustrative numerical examples, and heuristic interpretation of effect size analyses for neuropsychological researchers. Arch Clin Neuropsychol. 2001;16:653-67.

28. Camus V, Payoux P, Barre L, Desgranges B, Voisin T, Tauber C, et al. Using PET with 18F-AV-45 (florbetapir) to quantify brain amyloid load in a clinical environment. Eur J Nucl Med Mol Imaging. 2012;39:621-31. doi:10.1007/s00259-011-2021-8.

29. Landau SM, Breault C, Joshi AD, Pontecorvo M, Mathis CA, Jagust WJ, et al. Amyloid-beta imaging with Pittsburgh compound B and florbetapir: comparing radiotracers and quantification methods. J Nucl Med. 2013;54:70-7. doi:10.2967/ jnumed.112.109009.

30. Doraiswamy PM, Sperling RA, Coleman RE, Johnson KA, Reiman EM, Davis MD, et al. Amyloid- $\beta$ assessed by florbetapir F 18 PET and 18-month cognitive decline: a multicenter study. Neurology. 2012;79:1636-44. doi:10.1212/WNL.0b013e3182661f74.

31. Mintun MA, Larossa GN, Sheline YI, Dence CS, Lee SY, Mach RH, et al. [11C]PIB in a nondemented population: potential antecedent marker of Alzheimer disease. Neurology. 2006;67:446-52. doi:10. 1212/01.wnl.0000228230.26044.a4. 